\title{
Cognitive Neuropsychiatry and the Future of Diagnosis: a 'PC' Model of the Mind
}

\author{
BRUCE G. CHARLTON
}

\begin{abstract}
The current classification of the functional psychoses into three major syndromes of schizophrenia, depression and mania is, I believe, essentially misconceived. This 'syndromal' classification of psychiatric disease, while useful in the past and better than nothing (Kendall, 1975), now stands in the way of progress (Anon, 1985; Crow, 1986; Johnstone et al, 1988; Charlton, 1990; David, 1993). The categories of schizophrenia, depression and mania should be abandoned in favour of developing a cognitive nosology.
\end{abstract}

The syndromes of the 'functional psychoses' are defined in circular terms and on purely heuristic grounds, and they lack explanatory power (Healy, 1990). The diagnostic categories are notoriously imprecise, and depend upon the dominant pattern of symptoms (cross-sectionally and longitudinally) for most of their prognostic validity (e.g. Johnstone et al, 1988). Furthermore, the research programme of 'biological psychiatry' (based upon the search for causative lesions underlying the diagnostic syndromes) has failed to contribute significantly towards understanding aetiology or improving management of the functional psychoses (Charlton, 1990). This negative result may be interpreted as a powerful disconfirmation of the syndromal nosology.

I will argue that psychiatrists should consider adopting a cognitive nosology based upon a modern understanding of human psychological architecture and classifying patients in terms of lesions to information processing systems. The ultimate aim is to establish a new discipline: 'cognitive neuropsychiatry' (Frith, 1992; David, 1993; David \& Cutting, 1994). Because the concepts and language of cognitive psychology are unfamiliar to psychiatrists, I will outline a 'PC' (i.e. personal computer; rather than politically correct) model of the mind. According to this analogy, the functional psychoses are a consequence of deficits in inferential reasoning at the level of the mind's 'operating system'.

\section{The evolution of universal modular human psychology}

The period of evolution from hominid primate to modern Homo sapiens lasted roughly from 2.5 million to 100000 years ago (Konner, 1982; Barkow et al, 1992; Leakey, 1994). Physically, there has been little evolutionary change since that time - the gene pool is very similar everywhere and basic human anatomy, physiology and biochemistry is universal. Likewise, it is presumed that human psychological architecture has remained essentially unchanged, and also that the basic structure of the human mind is identical in all races and societies at every stage of economic development (Brown, 1991; Barkow et al, 1992).

Cognitive psychology conceptualises the human mind in terms of the steps in an information processing system which links input to output (Shallice, 1988). Cognitive processes are often described in the form of flow charts or decision trees: they are the computational protocols, algorithms and logical sequences by which perceptual data is framed, analysed and eventually converted into behaviour. ('Behaviour', in this sense, includes both external and internal physical changes, and subjective mental states such as rage or fear.)

Contemporary research has established that many specialised functions of human psychology are modular - information processing is divided into a finite number of discrete computational procedures, rather as the human body is divided into functionally independent organs to perform different physical procedures (Barkow et al, 1992). The relative independence (modularity) of cognitive functions is suggested by several lines of argument (Shallice, 1988). Firstly, humans have particular 'built in' functional aptitudes - most obviously the capacities involved in learning and using language (Barkow et al, 1992; Pinker, 1994). Secondly, clinicians have observed highly specific deficits in a context of otherwise normal function: for instance the discrete aphasias or agnosias seen in patients with brain lesions (Shallice, 1988). Thirdly, there are computational arguments which strongly suggest that complex and rapid information processing is impossible without subdivision of tasks into specialised and relatively autonomous subunits (Barkow et al, 1992; Pinker, 1994).

Human nature comprises a set of common psychological mechanisms (Shallice, 1988; Barkow et al, 1992; Pinker, 1994). As well as a 'general purpose' computational capacity, all (normal) humans share 
an identical set of cognitive modules capable of performing specialised computations. Each module was selected over an evolutionary timescale to solve survival problems and promote reproductive fitness in the Palaeolithic hunter-gatherer environment for which humans were 'designed' by natural selection (Konner, 1982; Barkow et al, 1992; Leakey, 1994).

If human nature is universal, how then do we explain the tremendous cultural and individual variety at the level of overt human behaviour? Simply because different inputs give rise to different outputs, and behaviour is therefore as varied as individual experience. So any diagnostic classification based upon overt behavioural outputs (such as the syndromal nosology) will be confounded by variation in inputs, and will not be cross-culturally valid (Healy, 1990). By contrast, the universal nature of evolved psychology means that a diagnostic scheme based upon cognitive processing would be universally applicable.

\section{A 'PC' model of the mind}

Perhaps the greatest barrier to the adoption of a cognitive neuropsychiatric perspective in clinical practice is the unfamiliarity and abstract nature of the language and concepts. In such circumstances a concrete analogy can be a helpful tool for thought. I will describe a model of the mind based upon information-processing in a personal computer (PC) in the hope that these machines will be familiar to most psychiatrists. But, however useful, any model must be treated with caution and will be misleading if pushed too far.

The human brain is conceived as a personal computer - a general purpose information processor (Table 1). A PC can perform many complex tasks by running a variety of specialised software (such as word processor, spread sheet, statistics or graphics packages), each of which produces a distinctive message (words, a graph etc.). An operating system (such as DOS or Windows) has the job of translating all these messages and assembling them into a unified display that can be understood by the computer operator: the essential role of the operating system is integration.

The analogous task of the human brain is to integrate messages from diverse cognitive modules into a form comprehensible to the conscious mind. We could imagine that the end product of integration is a cognitive printout $-\mathbf{a}$ continuously produced document where the messages from relevant software are assembled in linear sequence. The final step in the PC model is to 'make sense' of this printout. I will assume that the 'conscious mind' behaves like a computer operator who is inspecting a continuous
Table 1

A 'PC' model of the mind

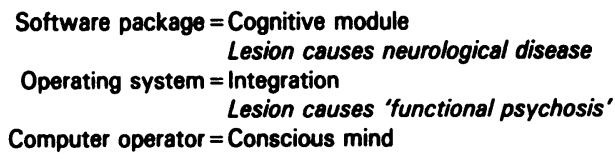

printout in order to generate appropriate behaviours. Furthermore, this computer operator has a sadly impoverished existence (!) and the printout is his only contact with the outside world.

A considerably more sophisticated and rigorous 'three-tier' model of the mind has recently been proposed by Sperber (1994). His description of input modules, first-order conceptual modules, and a second-order metarepresentational module seems compatible (mutatis mutandis) with my simple PC analogy.

I suggest that the characteristic symptoms of the functional psychoses are the consequence of a kind of 'software incompatibility' caused by damage to the operating system of the brain: in other words psychotic behaviours are the result of defective inference. In a 'pure' functional psychosis, the software modules are intact, but integration of their output messages is defective, so that the cognitive printout is incomplete, incorrectly sequenced or overinclusive. Psychotic behaviours are a consequence of the computer operator (the alert conscious mind) "trying to make sense" of a defective cognitive printout, and generating a response inappropriate to the original input. The computer operator does his best, but faced with garbled or incomplete information he draws the wrong inference: a reasonable response to an unreasonable cognitive printout.

An example of inappropriate integration of modules might tentatively be suggested. David (1994) has put forward the model of 'hyper-connection' between cognitive modules as a factor in some 'schizophrenic' symptoms. This would correspond to a faulty operating system mixing together software messages that ought to be kept apart. For instance, a 'reading' module reporting on a newspaper article might be wrongly integrated with a module for detecting threats to the organism, so that the cognitive printout blends impersonal information with inappropriate emotion. The 'perplexed' computer operator understands this to mean that the newspaper contains coded threats to the patient.

Neurological disorders, on the other hand, frequently display deficits in cognitive modules (Shallice, 1988) but without psychotic behaviours: integration and inferential processes are normal. Pure neurological 
disorders might be a consequence of lesioned software packages and an intact operating system. Because the operating system is normal, the problem is diagnosable by the computer operator. $\mathrm{He}$ is able to infer, for instance, that the word processor package has 'crashed', and he will make compensatory adjustments to behaviour.

If the above model is accurate in principle, it provides an explanation of the failure of biological psychiatry to discover a link between specific behavioural syndromes and damage to specific brain circuits (Charlton, 1990). Operating systems are defined functionally as protocols for bringing together cognitive outputs (i.e. drawing inferences), and the same operating systems can perform the same function on a variety of different PCs. An operating system such as Windows allows the same software package to run on a variety of structurally different machines. But if your operating system is at fault, it makes little sense to look for the problem among the circuits of your computer.

\section{The research agenda}

Research into the new discipline of cognitive neuropsychiatry demands a different methodology from that adopted in contemporary psychiatry. The necessary methods can be developed, despite certain logistical problems (Frith, 1992; David, 1993), from those already devised by cognitive neuropsychologists.

The cognitive methodology requires the use of single case studies (Caramazza, 1986; Shallice, 1988; Charlton, 1990; Charlton, 1991). Individual patients with defined cognitive lesions must be the unit of investigation rather than groups of patients defined in terms of a common diagnosis. Groups of patients are not suitable because cognitive models are qualitative phenomena, and qualities cannot be averaged (Charlton, 1995). Cognitive observations are qualitative tests of a theoretically-informed causal model, and are potentially replicable. Models should be as detailed and explicit as possible to enable potential refutation (Caramazza, 1986). Replication involves testing the model against individual cases - any of which may contradict, refine or replicate the model.

The ideal subject for identifying cognitive modules is a pure case (Caramazza, 1986; Shallice, 1988) a patient who demonstrates a single deficit affecting a specific cognitive mechanism against a background of normal function in all other cognitive systems. A pure neurological case would display the result of knocking-out a single cognitive module. A pure psychiatric case would have a full complement of intact modules, and largely normal inferential abilities, except for a single defect in inferential reasoning apparent on a specific class of functional tasks. Such information could, in context with other clinical findings and convergent and complementary evidence from other kinds of psychological study (Shallice, 1988), be used to help construct a model of the human 'operating system' (or 'first-order conceptual modular architecture' to use Sperber's terminology (1994)), and this model would then be tested against further cases.

Pure cases represent an ideal which can seldom be found in practice. However, research can also proceed by identifying dissociations between cognitive functions (Caramazza, 1986; Shallice, 1988). A neurological dissociation occurs when the experimental subject performs normally on one task and badly on another task: dissociation implies that the two tasks are mediated by different cognitive modules. Psychiatric dissociations occur when one type of inferential reasoning task is deficient and another type normal: this implies that the two tasks involve different parts of the operating system.

To return to the analogy: tasks requiring integration of word processing and graphics may be abnormal, but all other computational tasks are performed normally. The fact that this lesion can occur in isolation points to a functional separation within the operating system which may serve as the basis for a psychiatric diagnostic category (dyswordographia?).

The task for cognitive neuropsychiatry researchers is to assist in a (multidisciplinary) project to map the universal inferential architecture of the mind's operating system. The aim is to understand and describe the fundamental and universal pathways by which cognitive messages are recognised and integrated. Clinicians could make a major contribution by devising diagnostic tests of inference (or 'cognitive probes', see below) and incorporating them into the mental state examination. Ultimately, a psychiatric nosology may be constructed on the basis of specific 'lesions of inference' in the operating system.

\section{Implications for clinical practice}

Although it may be conceded that cognitive neuropsychiatry offers great potential as a research strategy, syndromal diagnosis is solidly established in clinical practice. Patients will, of course, continue to be referred to psychiatrists on the basis of severe behavioural abnormalities (symptoms and signs) and therapy should be based upon empirical effectiveness. The cognitive neuropsychiatric examination would be concerned with testing the patient's power of drawing inferences from presented stimuli in order to enable the psychiatrist to understand and explain the overt behaviours. This element of cognitive 
testing is not new to the psychiatric examination; the new approach would involve more a change of emphasis and diagnostic purpose.

Clearly, psychiatrists cannot use elaborate cognitive test batteries in routine clinical practice: such techniques would be reserved for difficult cases. However, psychiatrists already use brief evaluations of cognitive mechanisms in their normal historytaking. Tasks of cognitive inference could be verbal (e.g. interpretation of proverbs), visual (e.g. explaining uncaptioned comic strips), auditory (e.g. tape recordings played through headphones) logical (for instance problems of an "if this, then what?" form), or may use any other modality. Such tasks would share a strategy of using highly culturally specific but decontextualised information to reveal the presence and nature of qualitatively faulty inferences.

The goal of re-classifying the 'functional psychoses' in terms of lesions of the universal human operating system will, doubtless, take a great deal of research effort. The design, validation and interpretation of convenient cognitive probes, and a vocabulary for describing the inferential abnormalities which they seek, are major tasks ahead for cognitive neuropsychiatrists.

\section{Conclusions}

I have attempted to sketch the outlines of a cognitive nosology for the functional psychoses, including an indication of the possible shape of future research and clinical practice. If accepted, the cognitive model would have far-reaching consequences. Not least, there would have to be a re-definition of the disciplinary boundaries between modular lesions (neurological) and inferential lesions (psychiatric), which might reallocate a number of disorders or give rise to 'mixed' conditions.

Cognitive neuropsychiatric research has, to date, been fascinating (Ellis \& Young, 1990; Frith, 1992; David, 1993; David \& Cutting, 1994; David \& Howard, 1994), but must be regarded as preliminary. Previous work has too often been flawed by the lack of an evolutionary perspective (Barkow et al, 1992; McGuire et al, 1992), the employment of group studies rather than single cases, the failure to seek pure cases, or an adherence to the traditional syndromal diagnoses. The programme of cognitive neuropsychology is not, therefore, an 'off-the-peg' methodology which is simply waiting to be applied to psychiatry. Progress will depend upon researchers and clinicians observing their patients in a different way: honing their skills in the close observation of the inferential processes of individual patients. This effort would be worthwhile if, for the first time in many decades, psychiatrists might be in a position to contribute towards a fundamental understanding of human psychology.

Current practice already contains the seeds of a cognitive approach, but these are overshadowed by the continued dominance of the syndromal diagnoses. The first, and hardest, step is to break the habits of a century and stop talking about schizophrenia, mania and depression.

\section{Acknowledgements}

Several people have made important contributions to this paper, and I am very grateful for their comments and criticisms (although responsibility for the final result, especially any errors, is entirely my own). Particular thanks are due to Fraser Charlton, Tony David, Chris Frith, Janice Kay and Hamish McClelland.

\section{References}

ANON (editorial) (1985) Irritability in psychiatric nosologists. Lancet, $i i, 1123$.

BArkow, J. H., Cosmides, L. \& ToOBy, J. (eds) (1992) The Adapted Mind: Evolutionary Psychology and the Generation of Culture. New York: Oxford University Press.

Brown, D. E. (1991) Human Universals. New York: McGraw Hill. CaramazzA, A. (1986) On drawing inferences about the structure of normal cognitive systems from the analysis of patterns of impaired performance: the case for single patient studies. Brain and Cognition, 5, 41-66.

Chartton, B. G. (1990) A critique of biological psychiatry. Psychological Medicine, 20, 3-6.

(1991) Endocrine physiology and the value of case studies. Journal of Endocrinology, 130, 1-2.

(1995) Mega-trials: methodological issues and implications for clinical effectiveness. Journal of the Royal College of Physicians of London, 29, 96-100.

Crow, T. J. (1986) The continuum of psychosis and its implications for the study of the gene. British Journal of Psychiatry, 149, 419-429.

DAvid, A. (1993) Cognitive neuropsychiatry? Psychological Medicine, 23, 1-5.

- \& Cutting, J. C. (eds) (1994) The Neuropsychology of Schizophrenia. Hove: Lawrence Erlbaum Associates.

- \& HowARD, R. (1994) The experimental phenomenological approach to delusional memory in schizophrenia and late paraphrenia. Psychological Medicine, 24, 515-524.

- (1994) Dysmodularity: a neurocognitive model for schizophrenia. Schizophrenia Bulletin, 20, 249-253.

ELLIS, H. D. \& YounG, A. W. (1990) Accounting for delusional misidentification. British Journal of Psychiatry, 157, 239-248.

FrITH, C. D. (1992) The Cognitive Neuropsychology of Schizophrenia. Hove: Lawrence Erlbaum Associates.

Healy, D. (1990) The Suspended Revolution. London: Faber.

Johnstone, E. C., Crow, T. J., Frith, C. D., et al (1988) The Northwick Park 'functional' psychosis study: diagnosis and treatment response. Lancet, $i i, 118-125$.

Kendall, R. E. (1975) The Role of Diagnosis in Psychiatry. Oxford: Blackwell.

KONNER, M. (1982) The Tangled Wing: Biological Constraints on the Human Spirit. New York: Holt, Rinehart and Winston.

Leakey, R. (1994) The Origins of Humankind. London: Weidenfeld and Nicolson.

McGuire, M. T., MARKs, I., Nesse, R. M., et al (1992) Evolutionary biology: a basic science for psychiatry. Acta Psychiatrica Scandinavica, 86, 89-96. 
Pinker, S. (1994) The Language Instinct. Harmondsworth: Penguin.

Shallice, T. (1988) From Neuropsychology to Mental Structure. Cambridge: Cambridge University Press.
SPERBER, D. (1994) The modularity of thought and the epidemiology of representations. In Mapping the Mind: Domain Specificity in Cognition and Culture (eds L. A. Hirschfield \& S. A. Gelman). Cambridge: Cambridge University Press.

Bruce G. Chariton, MD, Department of Epidemiology and Public Health, University of Newcastle upon Tyne NE2 4 HH. Fax: 01912228211

(Received 2 February 1995, accepted 11 April 1995)

\section{Commentary}

\section{Brains, Biases, Deficits and Disorders \\ RICHARD P. BENTALL}

\begin{abstract}
Dissatisfaction with existing classifications of the psychotic disorders has led a number of authors to discuss new ways of addressing this problem. Some have advocated empirically-based alternatives to neoKraepelinian diagnostic categories (Liddle, 1987), others have advocated a dimensional approach to psychosis (Claridge, 1987), and others have suggested that syndromic classifications might be abandoned altogether in favour of research and treatment targeted at specific symptoms (Bentall et al, 1988). Charlton suggests a further 'cognitive neuropsychiatric' approach in which psychotic symptoms are seen as lesions in an operating system (analogous to that which controls the functions of a computer) which integrates the activities of intact cognitive modules. On this view, the future of psychiatric diagnosis will depend on the development of cognitive probes designed to identify lesions of inference, and research into the psychoses will largely follow the single-case study methodology advocated by neuropsychologists. While I have some sympathy with Charlton's aims (which share with the dimensional and symptomorientated approaches the attempt to link psychiatry to the understanding of normal psychological processes) I doubt whether the characterisation of human cognitive functioning on which he bases his arguments is sufficiently defensible to provide a sound basis for research. To illustrate my objections I will refer briefly to research into psychotic symptoms carried out by myself and other investigators.
\end{abstract}

\section{Modules, operating systems, cognitive deficits and cognitive biases}

Central to Charlton's arguments is the claim that the human mind consists of discrete, biologically implemented cognitive modules which are coordinated by an operating system. Although the hypothesis of modularisation is supported by neuropsychological research (Shallice, 1988), it is by no means clear that all important functions are organised in this way. Some functions, particularly those involved in general intelligence, may not be modularised (Anderson, 1983; Newell, 1990) as even advocates of the modular hypothesis have sometimes conceded (Fodor, 1983). Moreover, the idea of a central operating system which coordinates the activity of modules is reminiscent of the Cartesian theatre of the mind, and is implausible on both philosophical and scientific grounds (Dennett, 1991).

In fact, contemporary psychological research into the psychotic disorders tends to implicate specific psychological functions in specific symptoms, indicating that specific modules may be affected, at least in some cases. For example, I and several other authors (Hoffman, 1986; Bentall, 1990) have argued that auditory hallucinations result from the failure to attribute inner speech to self, which may reflect some kind of core reality-monitoring deficit. Frith (1992) has suggested that some psychotic symptoms may reflect a failure of meta-representation (specifically, the inability to represent the mental states of others).

Other cognitive abnormalities which have been implicated in psychotic disorders are best characterised as cognitive biases rather than cognitive deficits. Cognitive deficits are said to be present when there are disruptions of broad cognitive functions and are usually assessed using emotionally neutral test materials. It is natural to attribute such deficits to underlying neurophysiological abnormalities (Dennett, 1987). Cognitive biases, on the other hand, are said to be present when some forms of information are processed preferentially in comparison with others, 